\title{
Formación del banco de semillas durante la revegetación temprana de áreas afectadas por la minería en un bosque pluvial tropical del Chocó, Colombia
}

\author{
Hamleth Valois-Cuesta $^{1 *}$, Carolina Martínez-Ruiz ${ }^{2} \&$ Yorley Urrutia-Rivas ${ }^{1}$ \\ 1. Programa de Biología, Facultad de Ciencias Naturales, Universidad Tecnológica del Chocó, Cra. $22 \mathrm{~N}^{\circ} 18 \mathrm{~B}-10$, Barrio \\ Nicolás Medrano, Quibdó, Colombia; hamlethvalois@gmail.com, yorleurrutiad@gmail.com \\ 2. Escuela Técnica Superior de Ingenierías Agrarias, Instituto Universitario de investigación en Gestión Forestal \\ Sostenible UVa-INIA, Universidad de Valladolid, Av. Madrid, 50, 34071 Palencia, España; caromar@agro.uva.es \\ * Correspondence
}

Received 10-II-2016. Corrected 12-VII-2016. Accepted 17-VIII-2016.

\begin{abstract}
Soil seed bank formation during early revegetation of areas affected by mining in a tropical rain forest of Chocó, Colombia. Mining is one of the main economic activities in many tropical regions and is the cause of devastation of large areas of natural tropical forests. The knowledge of the regenerative potential of mining disturbed areas provides valuable information for their ecological restoration. The aim of this study was to evaluate the effect of age of abandonment of mines and their distance from the adjacent forest, on the formation of soil seed bank in abandoned mines in the San Juan, Chocó, Colombia. To do this, we determined the abundance and species composition of the soil seed bank, and the dynamics of seed rain in mines of different cessation period of mining activity ( 6 and 15 years), and at different distances from the adjacent forest matrix (50 and $100 \mathrm{~m}$ ). Seed rain was composed by five species of plants with anemocorous dispersion, and was more abundant in the mine of 6 years than in the mine of 15 years. There were no significant differences in the number of seeds collected at $50 \mathrm{~m}$ and $100 \mathrm{~m}$ from the adjacent forest. The soil seed bank was represented by eight species: two with anemocorous dispersion (common among the seed rain species) and the rest with zoochorous dispersion. The abundance of seeds in the soil did not vary with the age of the mine, but was higher at close distances to the forest edge than far away. During the early revegetation, the formation of the soil seed bank in the mines seems to be related to their proximity to other disturbed areas, rather than their proximity to the adjacent forest or the cessation activity period of mines. Therefore, the establishment of artificial perches or the maintenance of isolated trees in the abandoned mines could favour the arrival of bird-dispersed seeds at mines. However, since the soil seed bank can be significantly affected by the high rainfall in the study area, more studies are needed to evaluate management actions to encourage soil seed bank formation in mines of high-rainfall environments in the Chocó region. Rev. Biol. Trop. 65 (1): 393-404. Epub 2017 March 01.
\end{abstract}

Key words: forest away, abandoned mine, gold and platinum mining, passive revegetation, natural succession.

La minería a cielo abierto es una de las actividades antrópicas que genera mayores perturbaciones en los sistemas forestales naturales, ya que este tipo de intervención ocasiona fuertes cambios en la estructura y funcionamiento de dichos sistemas (Alday, Marrs, \& Martínez-Ruiz, 2010). La aplicación de programas de restauración ecológica de áreas afectadas por minería es una prioridad mundial, especialmente en aquellas regiones con alta biodiversidad y una clara vocación minera. La región del Chocó en Colombia es uno de los lugares más ricos del planeta en recursos biológicos (Rangel-Ch, 2004), así como en yacimientos de oro y platino, lo que ha hecho de esta región un punto de atracción para empresas mineras nacionales e internacionales, que ejecutan acciones de explotación 
con el consiguiente deterioro del patrimonio natural (Andrade-C, 2011). A pesar de la necesidad de mitigar los impactos que la minería ocasiona sobre los ecosistemas naturales, el conocimiento científico relativo a la aplicación de programas de restauración ecológica de áreas afectadas por minería en el Chocó es inexistente (pero ver Ayala, Mosquera, \& Murillo, 2008). Por lo tanto, no se cuenta con información que permita a los gestores aplicar medidas adecuadas para mitigar los impactos de la minería, a través de la puesta en marcha de modelos de restauración ecológica que faciliten el restablecimiento de la vegetación y sus propiedades emergentes.

El restablecimiento natural de la estructura y función de los sistemas forestales sometidos a algún tipo de perturbación, depende de numerosos factores y procesos ecológicos que se manifiestan a distintas escalas espaciales y temporales (Uhl, Buschbacher, \& Serrao, 1988; Aide \& Cavelier, 1994; Holl, 1999; Cubiña \& Aide, 2001), como la presencia de un flujo de entrada de semillas (lluvia de semillas) y la acumulación de éstas en el suelo (banco de semillas), que con el tiempo facilitan la revegetación natural de áreas perturbadas y contribuyen a mantener el equilibrio dinámico de los bosques naturales (Baker, 1989; Piudo \& Cavero, 2005). Una evaluación de la disponibilidad y magnitud de la lluvia y banco de semillas puede suministrar información vital para determinar la necesidad de introducir o no especies en áreas objeto de revegetación (Zhang, Shu, Lan, \& Wong, 2001; Walker, Walker, \& del Moral, 2007).

Desde el punto de vista de sus funciones ecológicas, el banco de semillas es un compartimento del ecosistema donde muchas especies aguardan el momento propicio para germinar y establecerse (Garwood, 1989; Tekle \& Bekele, 2000; Dalling, 2002; Álvarez-Aquino, Williams-Linera, \& Newton, 2005). Por su parte, la lluvia de semillas contribuye a la persistencia del banco del suelo en el tiempo, a través de un flujo constante o estacional de propágulos que llegan por diferentes formas de dispersión (Dalling, 2002). Conocer la abundancia, composición y dinámica del banco de semillas del suelo, así como del aporte de propágulos que entran a los sistemas vía lluvia de semillas, brinda información fundamental para entender el potencial regenerativo que tienen los sistemas forestales tras experimentar una perturbación por diversas actividades antrópicas (Parker, Simpson, \& Leck, 1989; Holl, 1999; Cardona \& Vargas, 2004).

Considerando que el restablecimiento de la cobertura vegetal y sus propiedades emergentes en áreas degradadas por minería a cielo abierto se ve afectado por factores y procesos naturales que se manifiestan de manera diferencial en el tiempo y en el espacio, se plantea el siguiente interrogante: ¿Cómo cambian el banco y la lluvia de semillas con el tiempo de abandono de las minas y la distancia de éstas a la matriz del bosque adyacente? La hipótesis de partida es que la abundancia, riqueza y composición de especies, tanto en el banco como en la lluvia de semillas, difieren conforme aumenta la edad de abandono de las minas y su lejanía a la matriz de bosque remanente.

El objetivo del presente estudio fue evaluar el efecto de la edad de abandono de las minas y su distancia al bosque adyacente, sobre la formación temprana del banco de semillas del suelo en las minas. Para ello, se determinó la abundancia, riqueza y composición de especies del banco de semillas del suelo y de la lluvia de semillas, así como la dinámica de esta última a lo largo de un año, en minas de distinto período de cese de la actividad minera, y la distancia a la matriz del bosque remanente en el San Juan, Chocó, Colombia.

\section{MATERIALES Y MÉTODOS}

Área de estudio: El trabajo se desarrolló en el corregimiento de Raspadura ( $5^{\circ} 13^{\prime} 17^{\prime \prime} \mathrm{N}$ - 76³8’37’ W), el cual pertenece al municipio de Unión Panamericana, Chocó (Colombia). Raspadura pertenece a la Subregión Central Norte del Chocó biogeográfico, la cual se caracteriza por presentar la mayor humedad disponible de la región, con precipitaciones de hasta de $11000 \mathrm{~mm}$ anuales, con un período 
de alta (mayo-agosto) y otro de baja precipitación (noviembre-febrero), temperatura promedio de $26{ }^{\circ} \mathrm{C}$ y humedad relativa mayor al $80 \%$. Esta zona presenta un tipo de roca sedimentaria transicional (Poveda-M, Rojas-P, Rudas-LI, \& Rangel-Ch, 2004).

El trabajo de campo se realizó en dos minas abandonadas, de 6 años $\left(5^{\circ} 13^{\prime} 37^{\prime \prime} \mathrm{N}\right.$

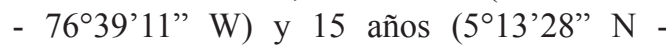
$76^{\circ} 38^{\prime} 55^{\prime \prime}$ W) tras el cese de la actividad minera. La edad post-aprovechamiento de cada mina fue determinada a partir de los registros históricos de uso de la tierra depositados en los archivos del Consejo Comunitario del Plan de Raspadura, y por medio de charlas con los dueños de los predios afectados. La minería aplicada fue a cielo abierto, haciendo uso de retroexcavadoras y motobombas que dejan como resultado un paisaje con apariencia desértica donde se observan grandes montículos de tierra lavada, arena y grava (Fig. 1A). La comunidad vegetal adyacente (bosque de referencia) a las minas estudiadas es propia de un bosque pluvial tropical (Poveda-M et al., 2004) en el que se pueden diferenciar tres estratos de vegetación (herbáceo, arbustivo y arbóreo), y dominancia de especies nativas de las familias Rubiaceae, Melastomataceae, Arecaceae, Piperaceae, Asteraceae, Fabaceae y Clusiaceae (Rangel-Ch \& Rivera-Díaz, 2004) como Pentaclethra macroloba, Psychotria longicuspis, Psychotria poeppigiana, Tococa guianensis, Palicourea seemannii, Anthurium aureum, Anthurium lancea, Cyclodium trianae, Ossaea bracteata, Ossaea spicata, Leandra chaetodon, Dieffenbachia plowmanii, Cyathea brunnescens y Dicranopygium cuatrecasanum (Valois-Cuesta, 2016).

Muestreo de lluvia de semillas: Se instalaron de manera permanente un total de 108 trampas de semillas en las dos minas seleccionadas de 6 y 15 años tras el cese de la actividad minera ( $\mathrm{N}=54$ en cada mina). Las trampas tenían un área de captura individual de $0.64 \mathrm{~m}^{2}(80 \times 80 \mathrm{~cm})$ y fueron construidas
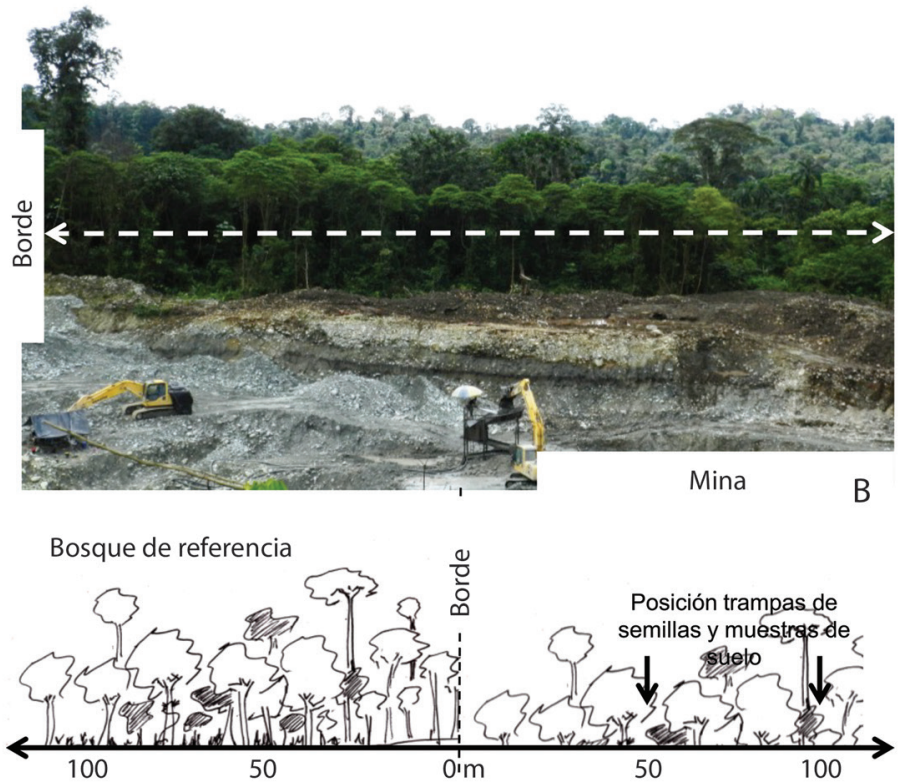

Fig. 1. Bosque afectado por minería en Raspadura, Unión Panamericana, Chocó, Colombia: (A) zona afectada por minería y (B) esquema para la toma de muestras de lluvia de semillas y banco del suelo en las minas.

Fig. 1. Forest affected by mining in Raspadura, Unión Panamericana, Chocó, Colombia: (A) area affected by mining and (B) scheme for sampling seed rain and soil seed bank in the mines. 
con una tela sintética de poliéster (luz $<1 \mathrm{~mm}$ ) que se encontraba suspendida a $80 \mathrm{~cm}$ de altura sobre el suelo, en una estructura armada con tubos de plástico (Stevenson \& Vargas, 2008). En cada mina, las trampas de semillas fueron distribuidas de manera perpendicular $(5 \mathrm{~m}$ de distancia entre trampas) a la matriz de vegetación adyacente, a 50 y $100 \mathrm{~m}$ de distancia (N $=27$ trampas por distancia y mina) respecto del borde bosque-mina (Fig. 1A y Fig. 1B). La retirada de las semillas capturadas por las trampas se realizó cada 15 días durante un año (de septiembre de 2013 hasta septiembre de 2014). Las muestras se conservaron en recipientes con etanol al $70 \%$ hasta su procesado (identificación y recuento).

Muestreo del banco de semillas: En diciembre 2014, en cada mina seleccionada se tomaron muestras de suelo hasta una profundidad de $5 \mathrm{~cm}$, con la ayuda de un barreno de $8 \mathrm{~cm}$ de diámetro $(\mathrm{N}=54$ por mina), tomando como puntos de muestreo los de ubicación de las trampas de semillas, a 50 y a $100 \mathrm{~m}$ de distancia desde el bosque adyacente $(\mathrm{N}=27$ muestras por distancia y mina). Estas muestras de suelo fueron trasladadas al laboratorio de Biología de la Universidad Tecnológica del Chocó, donde se secaron a temperatura ambiente para luego ser procesadas y separar las semillas del suelo con la ayuda de un tamiz (luz $<5 \mathrm{~mm}$ ).

Las muestras recolectadas en los experimentos de lluvia de semillas y del banco del suelo fueron analizadas en términos del número de semillas de cada especie identificada por trampa o muestra de suelo. La identificación taxonómica de las semillas se realizó por medio de confrontación con una colección de semillas de referencia, tomada en la zona durante el periodo de estudio, y con ejemplares depositados en la carpoteca del herbario Chocó de la Universidad Tecnológica del Chocó.

Tratamiento de los datos: El efecto de la edad de la mina (tiempo transcurrido tras el cese de la actividad minera), la distancia respecto a la fuente de semillas (bosque adyacente) y el momento de recogida de las trampas (a lo largo de un año de seguimiento) sobre la abundancia de la lluvia de semillas por muestreo, se evaluó mediante un ANOVA de medidas repetidas. El modelo incluyó la interacción entre los factores (edad y distancia), y el tiempo como una medida repetida. Este tipo de modelo también fue usado para evaluar los cambios de la lluvia de semillas en función de las especies y su interacción con la edad de la mina, la distancia al bosque adyacente y el momento del muestreo. En el caso del banco de semillas, el efecto de la especie, la edad de la mina y la distancia desde el bosque adyacente se analizó con un ANOVA de dos vías que incluyó la interacción entre los factores especie, edad y distancia. Adicionalmente, para analizar la contribución de las especies individuales a la lluvia de semillas y al banco del suelo, entre las diferentes distancias al bosque adyacente entre minas, se usó una ANOVA de una vía. En todos los casos donde los ANOVAs resultaron significativos, se usó posteriormente la prueba HSD de Tukey. Para los análisis, los datos fueron transformados a la raíz cuadrada de $(x+0.5)$ para ajustar su normalidad y homocedasticidad. Sin embargo, en el texto, cuadro y figuras los datos se muestran sin transformación (promedio \pm 2 error típico). Todos los análisis se llevaron a cabo en el entorno de programación $\mathrm{R}$ versión 2.15 (R Core Team, 2012), particularmente para los ANOVAs de medidas repetidas se usó el paquete "nlme" versión 3.1-120 (Pinehiro, Bates, DebRoy, \& Sarkar, 2015).

\section{RESULTADOS}

Lluvia de semillas: La cantidad de propágulos (número de semillas por trampa por muestreo) que llegaron a las minas vía lluvia de semillas varió con el período de abandono de la mina (ANOVA de medidas repetidas: $\left.\mathrm{F}_{1,104}=18.4, \mathrm{P}<0.0001\right)$, siendo mayor en la mina de 6 años $(24.8 \pm 2.16)$ que en la mina de 15 años $(15.3 \pm 1.84)$. No se encontraron diferencias estadísticamente significativas entre la cantidad de semillas recolectadas a $50 \mathrm{~m}$ (21.6 $\pm 2.16)$ у a $100 \mathrm{~m}(18.1 \pm 1.84)$ de distancia 
del bosque adyacente $\left(\mathrm{F}_{1,104}=1.83, \mathrm{P}=0.17\right)$. Sin embargo, las diferencias encontradas entre minas de distinta edad, fueron superiores a mayor distancia desde el bosque adyacente (interacción distancia $\times$ edad-mima: $\mathrm{F}_{1,104}=$ 34.5, $\mathrm{P}<0.0001$; Cuadro 1). La abundancia de la lluvia de semillas fluctuó a través del tiempo $\left(\mathrm{F}_{22,2228}=13.7, \mathrm{P}<0.0001\right)$ con variaciones significativas entre las minas de distintas edades (interacción tiempo $\times$ edad-mina: $\mathrm{F}_{21,2228}=$ $2.49, \mathrm{P}=0.0003$; Fig. $2 \mathrm{~A}$ ) y entre las diferentes distancias desde el bosque adyacente (interacción tiempo $\times$ distancia: $\mathrm{F}_{22,2228}=2.42, \mathrm{P}=$ 0.0001; Fig. 2B).

La lluvia de semillas estuvo compuesta por cinco especies de plantas, todas de dispersión anemócora (Cuadro 1). Estas especies se agruparon en tres géneros y tres familias y presentaron diferencias estadísticamente significativas en su contribución a la lluvia de semillas (ANOVA de medidas repetidas: $\mathrm{F}_{4,11670}=$ 1 851, P < 0.0001). Así, Andropogon bicornis (17.4 \pm 1.32$)$ fue la especie más abundante (HSD de Tukey, $\mathrm{P}<0.05$ ), seguida por Cespedesia spathulata (1.86 \pm 0.48$)$, Vernonia arborescens $(0.24 \pm 0.20)$, Vernonia braccharoides $(0.24 \pm 0.07)$ y Andropogon leocostachius $(0.12 \pm 0.05)$ con abundancias muy inferiores (HSD de Tukey, $\mathrm{P}>0.05$ ). Esta diferencia en la abundancia de semillas de estas especies se mantuvo con algunas variaciones a lo largo del año de estudio (interacción tiempo $\times$ especie: $\mathrm{F}_{88,11670}=6.79, \mathrm{P}<0.0001$; Fig. 3), así como con la edad de la mina (interacción edad-mina $\times$ especie: $\left.\mathrm{F}_{4,11670}=78.3, \mathrm{P}<0.0001\right)$ y la distancia al bosque adyacente (efecto de la interacción distancia $\times$ especie: $\mathrm{F}_{4,11670}=9.01, \mathrm{P}$ $<0.0001)$. Al respecto, la mayoría de especies contribuyeron más a la lluvia de semillas en la mina con 6 años de abandono y en distancias más cercanas al bosque adyacente (Cuadro 1).

Banco de semillas: El banco de semillas estuvo representado por ocho especies: dos de dispersión anemócora y seis de dispersión zoócora, tipo ornitócora y epizoócora (Cuadro 1). La cantidad de semillas (número de semillas por muestra de suelo) no varió con la edad de

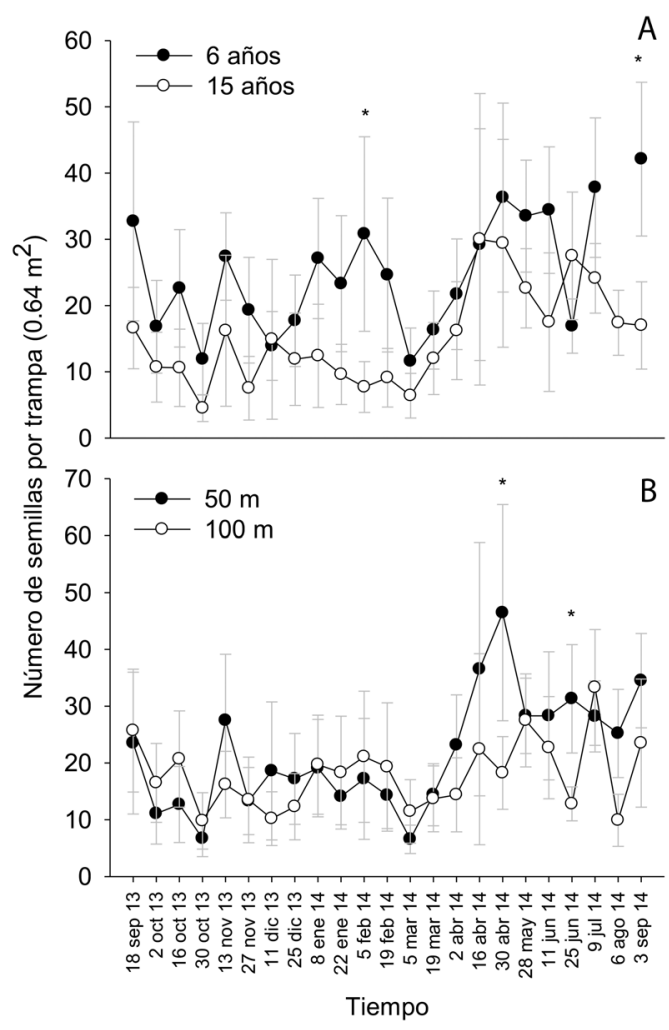

Fig. 2. Dinámica de la lluvia de semillas en minas abandonadas de distintas edades (A) y a diferentes distancias desde el bosque adyacente (B) en Raspadura, Unión Panamericana, Chocó, Colombia. Los datos representan la media \pm 2 error típico. Asteriscos (*) sobre los puntos indican diferencias estadísticamente significativas entre edades y distancias para algunos instantes temporales (HSD de Tukey, $\mathrm{P}<0.05$ ).

Fig. 2. Dynamic of seed rain in abandoned mines of different ages (A) and at different distances from the adjacent forest (B) in Raspadura, Unión Panamericana, Chocó, Colombia. Data as mean \pm 2 standard error. Asterisks (*) over the points indicate statistically significant differences between ages and distaces for some time instants (HSD Tukey, $\mathrm{P}<0.05)$.

la mina $(6$ años $=115 \pm 16.3 ; 15$ años $=115 \pm$ 18.8; ANOVA de dos vías: $\mathrm{F}_{1,104}=0.05, \mathrm{P}=$ 0.81 , pero sí con la distancia al bosque adyacente $\left(\mathrm{F}_{1,104}=31.6, \mathrm{P}<0.0001\right)$, siendo mayor a distancias próximas $(50 \mathrm{~m}=145 \pm 18.2)$ al bosque adyacente, que alejadas de éste $(100 \mathrm{~m}$ $=84.8 \pm 12.3)$. Además, la cantidad de semillas encontradas en el suelo a diferentes distancias desde el bosque adyacente no fue independiente 


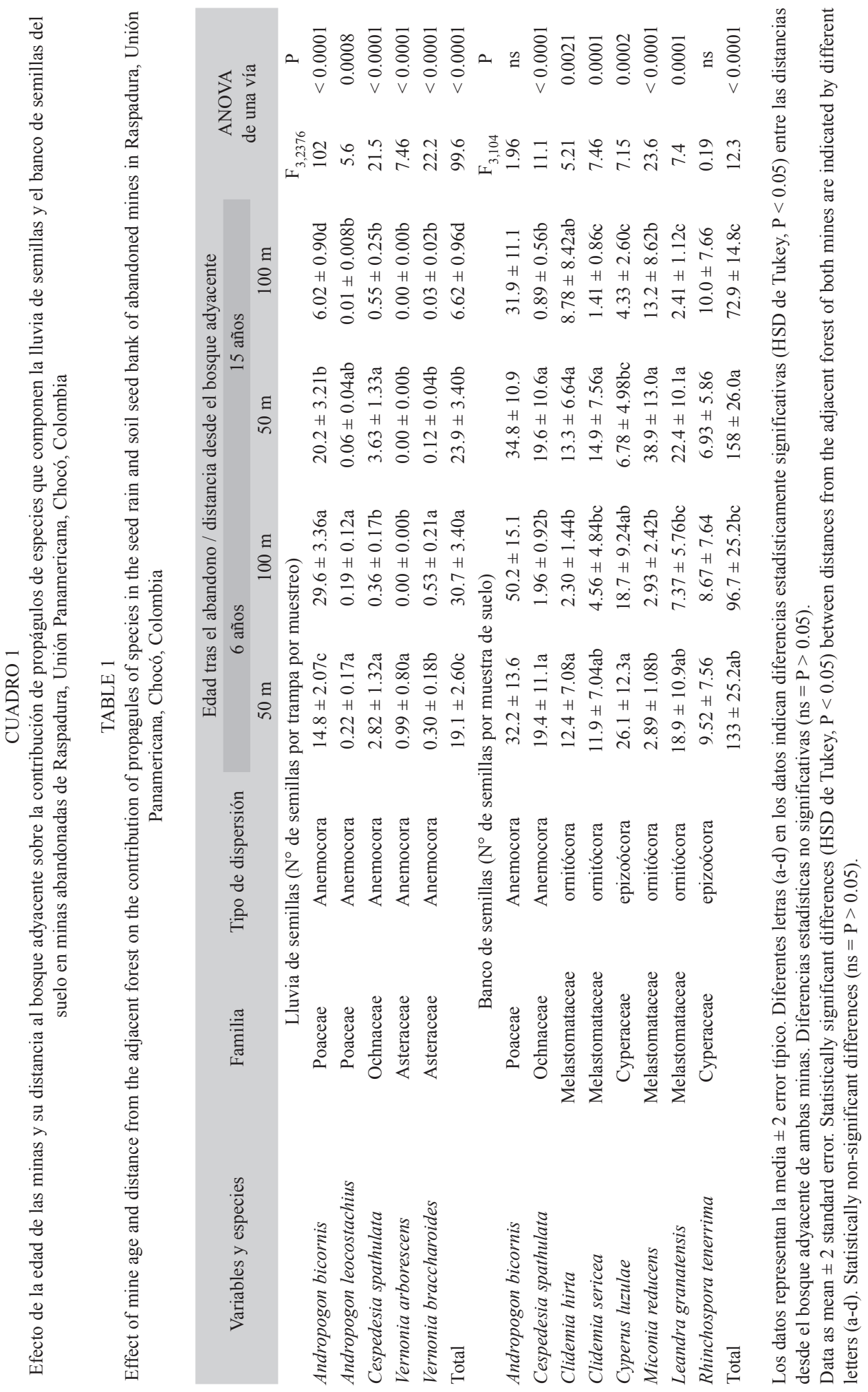




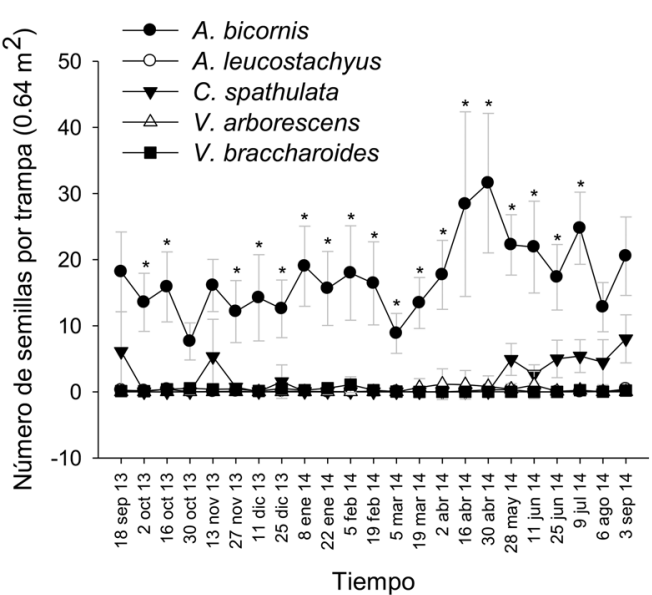

Fig. 3. Contribución de propágulos de las especies que conforman la lluvia de semillas en minas abandonadas de Raspadura, Unión Panamericana, Chocó, Colombia. Los datos indican la media \pm 2 error típico. Asteriscos (*) sobre los puntos indican diferencias estadísticamente significativas entre especies para diferentes instantes temporales (HSD de Tukey, $\mathrm{P}<0.05$ ).

Fig. 3. Contribution of propagules of the species that constitute the seed rain in abandoned mines in Raspadura, Unión Panamericana, Chocó, Colombia. Data as mean \pm 2 standard error. Asterisks (*) over the points indicate statistically significant differences among species for different time instants (HSD Tukey, $\mathrm{P}<0.05$ ).

de la edad de la mina (interacción distancia $\times$ edad: $\mathrm{F}_{1,104}=5.27, \mathrm{P}<0.02$ ), de modo que la distancia redujo con mayor magnitud la abundancia de semillas del banco en la mina de más edad tras el abandono (Cuadro 1).

Las especies encontradas también variaron significativamente en su contribución al banco de semillas $\left(\mathrm{F}_{7,839}=23.9, \mathrm{P}<0.0001\right)$; en este sentido, A. bicornis $(37.3 \pm 6.5)$ fue la especie mayormente representada (HSD de Tukey, $\mathrm{P}<$ 0.05), seguida por Cyperus luzulae (14.0 \pm 4.4$)$, Miconia reducens $(14.5 \pm 4.8)$, Leandra granatensis $(12.8 \pm 4.2)$, C. spathulata $=(10.4 \pm$ 4.2), Clidemia hirta (9.19 \pm 3.3$)$, Rhinchospora tenerrima $(8.8 \pm 3.6)$ y Clidemia sericea $(8.2 \pm$ 3.0) (HSD de Tukey, P > 0.05). Aquellas variaciones encontradas entre las especies, no fueron independientes de la edad de abandono de la mina (interacciones edad-mina $\times$ especies: $\left.\mathrm{F}_{7,839}=8.6, \mathrm{P}<0.0001\right)$ o de la distancia al bosque adyacente (interacción distancia $\times$ especie:
$\mathrm{F}_{7,839}=6.2, \mathrm{P}<0.0001$ ), en este sentido, $M$. reducens tuvo una mayor representatividad en la mina de 15 años, mientras que $C$. luzulae dominó más en la mina de 6 años, y las especies entre sí presentaron mayores diferencias a medida que se alejaban de la matriz del bosque adyacente (Cuadro 1).

\section{DISCUSIÓN}

La lluvia de semillas estuvo compuesta por tan sólo cinco especies de plantas, todas ellas de dispersión anemócora, siendo A. bicornis (Poaceae) la especie más abundante. Además la lluvia de semillas fue influida conjuntamente por la edad de abandono de las minas y la distancia al bosque adyacente, siendo mayor en la mina de 6 años que en aquella de 15 años, únicamente a los $100 \mathrm{~m}$ de distancia.

La riqueza de especies encontrada en las trampas de semillas fue muy baja si se compara con la elevada riqueza del bosque adyacente, en el que se registraron 232 especies de plantas vasculares (Valois-Cuesta, 2016). No obstante, otros estudios han mostrado patrones similares de baja riqueza de especies en la lluvia de semillas de áreas afectadas por intervención antrópica (Nepstad, Uhl, Serrao, \& de Souza, 1990; Aide \& Cavelier, 1994; Holl, 1999). Una de las posibles explicaciones puede estar en que la mayoría de las especies de arbustos y árboles que caracterizan los bosques tropicales son dispersadas por animales y, en particular, muchos dispersores de semillas evitan incursiones y/o forrajear en grandes espacios abiertos (característico de las minas) al ser más vulnerables a los depredadores, sobre todo si en los lugares abiertos no disponen de perchas o recursos alimentarios (Howe \& Smallwood, 1982; Estrada-Villegas, Pérez-Torres, \& Stevenson, 2007). En este sentido, la conservación de árboles remanentes en el seno de las minas podría jugar un papel importante en su revegetación por especies de plantas dispersadas por aves (Guevara, Purata, \& Van der Maarel, 1986; Guevara, Meave, Moreno-Casasola, \& Laborde, 1992; Guevara \& Laborde, 1993; Da Silva, Uhl, \& Murray, 1996; Estrada-Villegas et al., 2007). 
Desafortunadamente, toda la cobertura forestal es eliminada cuando las áreas son intervenidas para la extracción de oro y platino en la zona, limitando así las posibilidades de que semillas de algunas especies del bosque entren en las minas vía dispersión ornitócora. Por tanto, la disposición de perchas artificiales en las áreas afectadas por minería podría aumentar la lluvia de semillas dispersadas por animales, aunque otras barreras tales como la depredación post-dispersiva de las semillas, el tipo de sustrato predominante luego de la intervención minera y otros factores tensionantes puedan también limitar la regeneración del bosque en estas áreas críticas (Holl, 1999; Valois-Cuesta, 2016). En particular, algunas especies ruderales de porte arbustivo y/o arbóreo como $M$. reducens y $C$. spathulata, respectivamente, que crecen en las minas abandonadas y en bosques adyacentes (Valois-Cuesta, 2016), y que también fueron encontradas en el banco de semillas del suelo, podrían ser utilizadas para promover la entrada de semillas dispersadas por animales en áreas afectadas por minería en la zona.

En cuanto a la influencia de la edad de las minas y la distancia al bosque adyacente en la abundancia de la lluvia de semillas resulta difícil de explicar. En principio, cabría esperar mayor cantidad de semillas en trampas colocadas a menor distancia del bosque adyacente, como se ha encontrado en otros estudios (Álvarez-Buylla \& Martínez-Ramos, 1990; Cubiña \& Aide, 2001). Sin embargo, esta tendencia sólo se observa en la mina de mayor edad (15 años tras el abandono), mientras que la mina más joven (6 años) muestra una tendencia inversa. Posiblemente la explicación esté en el mecanismo de dispersión dominante entre las especies que constituyen la lluvia de semillas (todas son anemócoras) y a la incidencia y dirección del viento respecto a la posición y distancia entre las minas y las fuentes de propágulos, más que en diferencias de edad tras el abandono. En los estudios antes mencionados (Álvarez-Buylla \& Martínez-Ramos, 1990; Cubiña \& Aide, 2001) el tipo de especies predominantes en la lluvia de semillas fueron plantas leñosas de dispersión zoócora, que pueden provenir del bosque adyacente, mientras que en nuestras minas la lluvia de semillas está dominada por especies dispersadas por el viento que posiblemente provengan de otras áreas degradadas de regeneración temprana con abundancia de especies anemócoras y no del bosque adyacente. De hecho, Valois-Cuesta (2016) encontró que, tras 15 años de abandono, la composición florística de la comunidad vegetal de las minas difiere considerablemente de la composición florística del bosque adyacente, y está representada, en gran medida, por especies abundantes dispersadas por el viento que no se han encontrado en la comunidad vegetal del bosque adyacente, como es el caso de $A$. bicornis.

El banco de semillas del suelo estuvo compuesto por tan sólo ocho especies de plantas, dos presentes también en la lluvia de semillas y dispersadas por viento, y seis dispersadas por animales. De todas ellas la especie más abundante fue A. bicornis (Poaceae), al igual que en la lluvia de semillas. Además la abundancia de semillas del banco del suelo no varió con la edad de abandono de las minas, pero sí con la distancia al bosque adyacente, siendo mayor a distancias próximas al bosque, aunque sólo en la mina de más edad tras el abandono, la tendencia fue estadísticamente significativa.

La alta densidad de semillas de $A$. bicornis, tanto en la lluvia de semillas como en el banco del suelo, puede deberse a la facilidad que tienen sus semillas de llegar a las minas a través del viento y a la alta abundancia de individuos que esta especie presenta en zonas intervenidas circundantes, especialmente en aquellas perturbadas por minería (Valois-Cuesta, 2016). Esto explica por qué especies como C. hirta, $C$. luzulae, L. granatensis y $R$. tenerrima están menos representadas que $A$. bicornis en el banco de semillas de las minas (Cuadro 1), ya que a pesar de ser plantas comunes en el área de estudio (Valois-Cuesta, 2016), su llegada al seno de las minas puede verse limitada porque los animales dispersores de semillas evitan forrajear en grandes claros (como la minas) donde no disponen de perchas para su descanso y/o alimentación (Howe \& Smallwood, 1982; Estrada-Villegas et al., 2007). El pequeño 
tamaño del banco de semillas, tanto en abundancia de semillas como en riqueza de especies es frecuente en zonas tropicales si se compara con ecosistemas temperados (Skoglund, 1992; Kalamees, Püssa, Zobel, \& Zobel, 2012), y se explica por el mayor riesgo de pérdida de semillas por alta mortalidad (p.e. depredación, patógenos, fuego, etc.), o por presentar germinación intermitente relacionada con las efímeras condiciones favorables (p.e. disponibilidad de agua o calidad del sustrato) (Skoglund, 1992; Baskin \& Baskin, 2014).

Desde el punto de vista de la restauración ecológica, la menor importancia del banco de semillas en zonas tropicales hace que la eficacia del uso de suelo para recubrir estériles de mina en zonas tropicales sea menor que en ecosistemas temperados, en los que el importante papel que el banco de semillas del suelo juega en la dinámica de la vegetación (Garwood, 1989; Vyvey, 1989; Skoglund, 1992) ha incentivado el uso de suelos "naturales", en el recubrimiento de los estériles de mina o suelos degradados, como fuente natural de semillas capaz de favorecer la revegetación (Skoglund, 1992; Parrotta \& Knowles, 2001; Zhang et al., 2001), incluso sin la necesidad de emplear medidas adicionales (Bradshaw \& Chadwick, 1980; Bradshaw, 1997, 2000). Desde otro punto de vista, dado que los suelos afectados por la minería experimentan una reducción sustancial de las propiedades físicas, químicas y biológicas que sustentan el crecimiento de la vegetación (Valois-Cuesta, 2016), si se compara con los suelos en bosques de referencia (Quinto \& Hurtado, 2014), una manera de promover la revegetación temprana de las minas, podría ser la aplicación de enmiendas orgánicas que mejoren y/o normalicen las condiciones de suelo (Valois-Cuesta, 2016), a fin de generar escenarios que faciliten la germinación de las semillas que llegan a las minas y se establecen en el banco del suelo.

La mayor abundancia de semillas en el banco del suelo encontrada a menor distancia del bosque adyacente concuerda con la tendencia observada por otros autores. En concreto, Álvarez-Buylla y Martínez-Ramos
(1990) encuentran un descenso en la densidad de plántulas a mayor distancia del borde del bosque adyacente, demostrando que pocas semillas son dispersadas hacia el seno del área en regeneración, aun cuando se den las condiciones para que ocurra la dispersión. Cubiña y Aide (2001) encuentran diferencias en la abundancia de plántulas germinadas a partir de muestras de suelo recolectadas a diferentes distancias desde el bosque adyacente; más del $99 \%$ de las plántulas germinaron en muestras recolectadas dentro de los primeros $4 \mathrm{~m}$ de distancia respecto al bosque adyacente. Estos mismos autores (Álvarez-Buylla \& MartínezRamos, 1990; Cubiña \& Aide, 2001) sugieren también la posibilidad de que la menor acumulación de semillas a ciertas distancias desde el bosque adyacente se deba a la pérdida de viabilidad o altos niveles de depredación postdispersión. En el contexto de alta precipitación en el Chocó, es posible que los patrones de baja abundancia de semillas en el banco del suelo, especialmente a distancias alejadas del bosque de referencia, se deba a procesos de lavado por escorrentía superficial. En esta región donde los eventos de lluvia son intensos durante casi todo el año ( $>4000 \mathrm{~mm}$ año ${ }^{-1}$; Poveda-M et al., 2004) es posible que las semillas que llegan desde el bosque con periodos de latencia largo no germinen en la mina porque son lavadas hacia fuera del sistema. Aunque muchos estudios sobre sucesión han demostrado que el bosque se recupera en terrenos abandonados, también se ha indicado que sólo un pequeño grupo de las especies del bosque contribuirá al proceso de recuperación durante los primeros años de sucesión (Cubiña \& Aide, 2001; Alday, Pallavicini, Marrs, \& Martínez-Ruiz, 2011). El fuerte efecto de la distancia desde el bosque adyacente sobre la abundancia y riqueza de semillas del banco del suelo probablemente esté también relacionado con la disponibilidad y comportamiento de los dispersores, ya que la distribución espacial de las semillas durante el proceso de dispersión depende del hábito de alimentación y nidificación de los dispersores o de las costumbres de los animales de evitar lugares abiertos (Dalling, 2002). 
Se puede concluir que la entrada de propágulos a las minas vía lluvia de semillas puede estar más relacionada con la cercanía a otras áreas perturbadas, donde la abundancia de especies anemócoras es alta, que con la edad de abandono de las minas o su distancia desde el bosque de referencia. Al respecto, el establecimiento de perchas o bosquetes artificiales en el seno de las minas podría promover la recuperación vegetal de minas abandonadas con especies de dispersión zoócora (particularmente dispersadas por aves), cuya representación en el banco del suelo, especialmente en zonas cercanas al bosque adyacente, resulta interesante. No obstante, dado que el banco de semillas del suelo se puede ver afectado por los eventos de alta precipitación que imperan en la región del Chocó, es necesario idear medidas de control de la erosión en estas áreas críticas para promover la retención de semillas en el suelo. Por lo anterior, se recomienda emprender futuras investigaciones que evalúen experimentalmente el efecto de perchas artificiales y de algunas medidas de control de la erosión del suelo sobre la formación del banco de semillas, y en ese mismo contexto, determinar cómo la aplicación de enmiendas orgánicas al suelo puede facilitar la germinación del banco de semillas y el establecimiento de plantas en áreas afectadas por minería bajo un escenario de alta pluviosidad, como sucede en la región del Chocó. En todo caso, la restauración ecológica de las zonas afectadas por la minería dependerá de una adecuada gestión de territorio que involucre aspectos, no solo ecológicos, sino también sociales, culturales y económicos (Muñoz et al., 2012; Muñoz, Basanta, DíazVizcaíno, Reyes, \& Casal, 2014).

\section{AGRADECIMIENTOS}

A la comunidad del corregimiento Raspadura, Unión Panamericana, Chocó (Colombia) por su hospitalidad y apoyo. A S Eccehomo por su acompañamiento. A $\mathrm{K} \mathrm{J}$ Herrera, J F Lizarda, Z Quinto y K Sánchez por su ayuda en el trabajo de campo. A tres revisores anónimos por sus valiosos comentarios.
Este trabajo fue financiado por la Universidad Tecnológica del Chocó (UTCH) (convocatoria 2013-2014); por la UTCH y el Instituto de Investigaciones Ambientales del Pacífico (IIAP) a través del Proyecto "Desarrollo de Herramientas de Gestión para el Posicionamiento de la Biodiversidad como Fuente de Bienestar Social y Ambiental en el Chocó, Occidente Colombiano (BIOCHOCÓ) (Código BPIN 2013000100191); y por la Fundación Carolina en convenio con la UTCH y la Universidad de Valladolid (UVa) a través de una beca de doctorado asignada a $\mathrm{H}$ Valois.

\section{RESUMEN}

La minería es una de las principales actividades económicas en muchas regiones tropicales y también es la causa de la devastación de amplias superficies de bosques tropicales naturales. El conocimiento del potencial regenerativo de las áreas alteradas por minería es vital para la restauración ecológica de estas áreas críticas. El objetivo del presente trabajo fue evaluar el efecto de la edad de abandono de las minas y su distancia al bosque adyacente sobre la formación del banco de semillas del suelo en minas abandonadas en el San Juan, Chocó, Colombia. Para ello, se determinó la abundancia y composición de especies del banco de semillas, así como la dinámica de la lluvia de semillas, en minas de distinta edad (6 y 15 años), tras el cese de la actividad minera, y distancia a la matriz del bosque adyacente $(50$ y $100 \mathrm{~m})$. La lluvia de semillas estuvo compuesta por cinco especies de plantas, todas de dispersión anemócora, y fue mayor en la mina de 6 años que en la de 15 . No hubo diferencias significativas en la cantidad de semillas recolectadas a $50 \mathrm{~m}$ y $100 \mathrm{~m}$ de distancia del bosque adyacente. El banco de semillas estuvo representado por ocho especies: dos de dispersión anemócora (comunes a la lluvia de semillas) y el resto de dispersión zoócora. La abundancia de semillas en el suelo no varió con la edad de la mina, pero fue mayor a distancias próximas al bosque que alejadas de este. Durante la regeneración temprana, la formación del banco de semillas en el seno de las minas parece estar relacionada con la cercanía a otras áreas alteradas más que con su proximidad al bosque adyacente o la edad de las minas. Al respecto, el establecimiento de perchas artificiales o conservación de árboles remanentes en el seno de las minas podrían promover la entrada de semillas dispersadas por aves. No obstante, dado que el banco de semillas del suelo puede verse afectado por la alta precipitación de la zona, se recomienda promover estudios que valoren medidas encaminadas a favorecer la formación del banco de semillas del suelo de las minas en ambientes de alta pluviosidad como en la región del Chocó. 
Palabras clave: distancia del bosque, mina abandonada, minería auroplatinífera, revegetación pasiva, sucesión natural.

\section{REFERENCIAS}

Aide, T. M., \& Cavelier, J. (1994). Barriers to lowland forest tropical restoration in the Sierra Nevada de Santa Marta, Colombia. Restoration Ecolology, 2, 219-229.

Alday, J. G., Marrs, R. H., \& Martínez-Ruiz, C. (2010). The importance of topography and climate on shortterm vegetation of coal wastes in Spain. Ecological Engineering, 36, 579-585.

Alday, J. G., Pallavicini, Y., Marrs, R. H., \& MartínezRuiz, C. (2011). Functional groups and dispersal strategies as guides for predicting vegetation dynamics on reclaimed mines. Plant Ecology, 112, 1759-1775.

Álvarez-Aquino, C., Williams-Linera, G., \& Newton, A. C. (2005). Disturbance effects on the seed bank of Mexican cloud forest fragments. Biotropica, 37, 337-342.

Álvarez-Buylla, E. M., \& Martínez-Ramos, M. (1990). Seed bank versus seed rain in the regeneration of a tropical pioneer tree. Oecologia, 84, 314-325.

Andrade-C, G. (2011). Estado del conocimiento de la biodiversidad en Colombia y sus amenazas. Consideraciones para fortalecer la interacción ciencia-política. Revista de la Academia Colombiana de Ciencias Exactas, Fisicas y Naturales, 35, 491-507.

Ayala, J. H., Mosquera, J., \& Murillo, W. I. (2008). Evaluación de la adaptabilidad de la acacia (Acacia mangium Wild), y bija (Bixa orellana) en áreas degradadas por la actividad minera aluvial en el Chocó biogeográfico, Condoto, Chocó, Colombia. Bioetnia, $5,115-123$.

Baker, H. G. (1989). Some aspects of the natural history of the seed banks. In M. A. Leck, V. Parker, \& R. L. Simpson (Eds.), Ecology of soil seed banks (pp. 9-21). San Diego California: Academic Press INC.

Bradshaw, A. D. (1997). Restoration of mined lands using natural processes. Ecological Engineering, $8,255-269$

Baskin, C. C., \& Baskin, J. M. (2014). Seeds: ecology, biogeography and evolution of dormancy and germination ( $2^{\text {st }}$ ed). San Diego, CA: Academic Press.

Bradshaw, A. D. (2000). The use of natural processes in reclamation - advantages and difficulties. Landscape and Urban Planning, 51, 89-100.

Bradshaw, A. D., \& Chadwick, M. J. (1980). The Restoration of Land ( $1^{\text {st }}$ ed.). Oxford: Blackwell Scientific Publications.
Cardona, C. A., \& Vargas, O. R. (2004). Plántulas procedentes del banco de semillas germinable de un bosque subandino. Pérez-Arbelaezia, 15, 113-149.

Cubiña, A., \& Aide, T. M. (2001). The effect of distance from forest edge on seed rain and soil seed bank in a tropical pasture. Biotropica, 33, 260-267.

Dalling, J. W. (2002). Ecología de semillas. En M. R. Guariguata \& G. H. Kattan (Eds.), Ecología y conservación de bosques neotropicales (pp. 345-375). Cartago, Costa Rica: Ediciones LUR.

Da Silva, J. M. C., Uhl, C., \& Murray, G. (1996). Plant succession, landscape management, and the ecology of frugivorous birds in abandoned Amazonian pastures. Conservation Biology, 10, 491-503.

Estrada-Villegas, S., Pérez-Torres, J., \& Stevenson, P. (2007). Dispersión de semillas por murciélagos en un borde de bosque montano. Ecotropicos, 20, 1-14.

Garwood, N. C. (1989). Tropical soil seed banks: a review. In M. A. Leek, V. T. Parker, \& R. L. Simpson (Eds.), Ecology of soil seed banks (pp. 149-209). San Diego, California: Academic Press INC.

Guevara, S., Purata, S. E., \& Van der Maarel, E. (1986). The role of remnant forest trees in tropical secondary succession. Vegetatio, 66, 77-84.

Guevara, S., Meave, J., Moreno-Casasola, P., \& Laborde, J. (1992). Floristic composition and structure of vegetation under isolated trees in Neotropical pastures. Journal of Vegetation Science, 3, 655-664.

Guevara, S., \& Laborde, J. (1993). Monitoring seed dispersal at isolated standing trees in tropical pastures: consequences for local species availability. Vegetatio, 107, 319-338.

Holl, K. D. (1999). Factors limiting tropical rain forest regeneration in abandoned pasture: seed rain, seed germination, microclimate, and soill. Biotropica, 31, 229-242.

Howe, H. F., \& Smallwood, J. (1982). Ecology of seed dispersal. Annual Review of Ecology and Systematics, 13, 201-228.

Kalamees, R., Püssa, K., Zobel, K., \& Zobel, M. (2012). Restoration potential of the persistent soil seed bank in successional calcareous (alvar) grasslands in Estonia. Applied Vegetation Science, 15, 208-218.

Muñoz, A., Basanta, M., Díaz-Vizcaíno, E., Reyes, O., \& Casal, M. (2014). Land use changes effect on floristic composition, diversity and surface occupied by Erica ciliaris and Erica tetralix heathlands of NW Spain. Land Degradation \& Development, 25, 532-540.

Muñoz, A., Pesqueira, X. M., García-Duro, J., Álvarez, R., Reyes, O., \& Casal, M. (2012). Conservation of Erica ciliaris and Erica tetralix communities: the role 
of land management and physical environment on the composition, species richness and presence of endemic taxa. Wetlands, 32, 693-703.

Nepstad, D., Uhl, C., Serrao, E. A., \& de Souza, E. A. (1990). Surmounting barriers to forest regeneration in abandoned, highly degraded pastures: a case study from Paragominas, Pará, Brazil. In A. B. Anderson (Ed.), Alternatives to deforestation: steps towards sustainable use of the Amazon rain forest (pp. 215229). New York: Columbia University Press.

Parker, V., Simpson, R., \& Leck, M. (1989). Pattern and process in the dynamics of seed banks. In M. A. Leck, V. Parker, \& R. L Simpson (Eds.), Ecology of soil seed banks (pp. 367-384). San Diego, California: Academic Press INC.

Parrotta, J. A., \& Knowles, O. H. (2001). Restoring tropical forests on lands mined for bauxite: Examples from the Brazilian Amazon. Ecological Engineering, 17, 219-239.

Piudo, M. J., \& Cavero, R. Y. (2005). Banco de semillas: comparación de metodologías de extracción, de densidad y de profundidad de muestreo. Publicaciones de Biología, Universidad de Navarra, Serie Botánica, $16,71-85$.

Pinehiro, J., Bates, D., DebRoy, S., \& Sarkar, D. (2015). NLME: Linear and nonlinear mixed effects models. Recuperado de http://cran.r-project.org/web/packages/nlme/nlme.pdf

Poveda-M, C., Rojas-P, C. A., Rudas-LI, A., \& Rangel-Ch, J. O. (2004). El Chocó biogeográfico: ambiente físico. En J. O. Rangel-Ch (Ed.), Colombia diversidad biótica IV, El Chocó biogeográfico/Costa Pacífica (pp. 1-22). Bogotá, Colombia: Universidad Nacional de Colombia-Conservación Internacional.

R Core Team (2012). R: A language and environment for statistical computing. Vienna, Austria: R Foundation for Statistical Computing. Recuperado de http:// www.R-project.org/

Rangel-Ch, J. O. (2004). Colombia diversidad biótica IV. El Chocó Biogeográfico/Costa Pacífica. Bogotá, Colombia: Universidad Nacional de Colombia, Conservación Internacional.

Skoglund, J. (1992). The role of seed banks in vegetation dynamics and restoration of dry tropical ecosystems. Journal of Vegetation Science, 3, 357-360.

Stevenson, P. R., \& Vargas, I. N. (2008). Sample size and appropriate design of fruit and seed traps in tropical forests. Journal of Tropical Ecology, 24, 95-105.

Tekle, K., \& Bekele, T. (2000). The role of soil seed banks in the rehabilitation of degraded hills lopes in Southern Wello, Ethiopia. Biotropica, 32, 23-32.

Uhl, C., Buschbacher, R., \& Serrao, E. A. (1988). Abandoned pastures in eastern Amazonia. I. Patterns of plant succession. Journal of Ecology, 73, 663-681.

Valois-Cuesta, H. (2016). Sucesión primaria y ecología de la revegetación de selvas degradadas por minería en el Chocó, Colombia: bases para su restauración ecológica (Tesis doctoral). Universidad de Valladolid, España.

Vyvey, Q. (1989). Bibliographical review on buried viable seed in the soil. Excerpta Botanica Section, 27, 1-52.

Walker, L. R., Walker, J., \& del Moral, R. (2007). Forging a new alliance between succession and restoration. In L. R. Walker, J. Walker, \& R. J. Hobbs (Eds.), Linking restoration and ecological succession (pp. 1-18). New York: Springer.

Zhang, Z. Q., Shu, W. S., Lan, C. Y., \& Wong, M. H. (2001). Soil seed bank as an input of seed source in revegetation of lead/zinc mine tailings. Restoration Ecology, 9, 378-385. 